\title{
Arbitrary Spectral Synthesis and Waveform Generation with HiBi Fiber Loop Mirrors
}

\author{
Haijun He, Li-Yang Shao, Chao Wang, Bin Luo, Xihua Zou, Xinpu Zhang, Wei Pan, and Lianshan Yan
}

\begin{abstract}
An arbitrary spectral synthesis scheme with parallel-connecting high-birefringence fiber loop mirrors (HiBi-FLMs) based on Fourier synthesis theory has been proposed and demonstrated. Three typical spectra of triangle, rectangle and sawtooth shape have been synthesized by implementing only four HiBi-FLMs. The experimental results are in good agreement with theoretical simulations with a goodness of fit of 0.9565 . Furthermore, higher precise optical spectrum with narrower bandwidth can be obtained by adopting longer polarizationmaintaining fiber and more sections of HiBi-FLMs. Besides, a typical application of arbitrary waveform generation has been implemented. By incorporating with frequency-totime mapping, triangle- and sawtooth-shaped electrical pulses with repetition rate of $1 \mathrm{GHz}$ and pulse width of $\sim 860$ ps have been generated.
\end{abstract}

Index Terms-Fiber optics, Polarization-maintaining Fibers, Sagnac effect, Arbitrary spectral synthesis, Arbitrary waveform generation.

\section{INTRODUCTION}

$\mathrm{O}$ ptical fiber loop mirror with high-birefringence fiber has attracted much attention due to its unique advantages of simple design, ease of manufacture, low insertion loss and good compatibility with other systems. In the past few decades, high-birefringence fiber loop mirror (HiBi-FLM) has been studied intensively and widely applied in many fields such as fiber comb filters [1-3], all-optical signal processing [4,5], multi-wavelength fiber lasers [6], gain-flattening erbium-doped fiber amplifier (EDFA) [7], optical fiber sensors [8,9] and photonic generation of arbitrary microwave waveforms $[10,11]$.

Manuscript received XX, 2018; accepted XX, 2018. Date of publication XX, 2018; date of current version XX, 2018. This research is supported by the International Science and Technology Cooperation Program of China (2014DFA11170), National Natural Science Foundation of China (61475128), Key Project of Sichuan Provincial Science and Technology Plan (2017GZ0091), Doctoral Innovation Fund Program of Southwest Jiaotong University (D-CX201718). (Corresponding author: Li-Yang Shao)

H. He, L. Shao, B. Luo, X. Zhang, X. Zou, W. Pan, and L. Yan are with Center for Information Photonics and Communications, Southwest Jiaotong University, Chengdu, 611756, China (e-mail: haijunhe@my.swjtu.edu.cn; lyshao@home.swjtu.edu.cn;

zouxihua@home.swjtu.edu.cn;

wpan@swjtu.edu.cn; 1syan@home.swjtu.edu.cn).

L. Shao is now with the Department of Electrical and Electronic Engineering, Southern University of Science and Technology, Shenzhen 518055, China (e-mail: shaoly@ sustc.edu.cn).

C. Wang is with School of Engineering and Digital Arts, University of Kent, Canterbury, CT27NT, U.K. (e-mail: c.wang@kent.ac.uk).
Especially, the HiBi microfiber and silicon photonics make it tremendous potential for manufacturing micro photonic devices $[12,13]$.

For photonic arbitrary waveform generation, a HiBi-FLM usually acts as a spectrum shaper, which is followed by frequency-to-time mapping (FTTM) in a dispersion element to generate arbitrary microwave waveforms [10]. Therefore, the spectrum shaper is a key component in FTTM-based photonic arbitrary waveform generation. Although a single-section HiBi-FLM [10], cascaded multi-section HiBi-FLMs [14,15] and FLM incorporating a chirped fiber Bragg grating [16] were adopted, all of these approaches are only capable of generating some specific spectrum shapes.

In this work, we propose and demonstrate a novel arbitrary spectral synthesis method with parallel-connecting HiBi-FLMs based on Fourier synthesis theory. Although Fourier transform synthesis has been explored in conventional optical arbitrary waveform generation (OAWG) by tailoring the individual frequency comb lines of ultrashort optical pulses [17], there are few reports about direct optical spectrum shaping (especially for arbitrary optical spectrum). Besides, the optical spectrum of the conventional OAWG is composed by many discrete spectral lines. Different from the conventional OAWG, the proposed parallel-connecting HiBi-FLMs can synthesize continuous optical spectrum with arbitrary shape. To verify the feasibility of the proposed technique, four parallel HiBi-FLMs have been constructed to synthesize three typical spectra including triangle, rectangle and sawtooth shapes. The goodness of fit between the test results and theoretical simulations reaches up to 0.9565 . Besides, temporal arbitrary waveforms can be generated incorporating FTTM conversion. Triangle- and sawtooth-shaped temporal waveforms have been generated in experiment. Therefore, the proposed method can synthesize arbitrary optical spectra and generate arbitrary temporal waveform as well. Except for the photonic arbitrary waveform generation, the proposed optical spectrum synthesis provides a promising solution for customized optical filtering, such as bandpass filter, edge filter and other elements.

\section{PRINCIPLE OF THE ARBITRARY SPECTRAL SYNTHESIS}

The conventional HiBi-FLM includes a broadband light source, a 3-dB single mode coupler, a polarization controller (PC) and a piece of polarization-maintaining fiber (PMF) [8]. The transmission function of the HiBi-FLM is $T=A / 2[1+\cos (\varphi)] . \varphi=2 \pi B L / \lambda$ is the phase, $B$ is the birefringence of PMF, $L$ is the length of the PMF, $\lambda$ is the wavelength, $A$ represents the extinction coefficient, which can 
be tuned from 0 to 1 through adjusting PC. Because of $\lambda=c / f$, the phase term can be rewritten as $\varphi=2 \pi B L f / c$ ( $f$ is the frequency of light, $c$ is the velocity of light in vacuum). Therefore, the transmission function of angular frequency $(\omega$, $\omega=2 \pi f=2 \pi c / \lambda)$ can be described as $T(w)=A / 2[1+\cos (t w)], t$ equals to $B L / c, B L$ represents fiber's property. Once the type and length of PMF are determined, $t$ is unchanged when the fiber loop exists in a constant environment.

According to previous reports, the phase $\varphi^{\prime}$ can be derived from $\varphi$ when PMF is tuned by strain, temperature and torsion $[8,9]$. So, the derived phase can be rewritten as $\varphi^{\prime}=\varphi+\Delta \varphi(\varphi$ is much larger than $\Delta \varphi)$. Replacing $\varphi$ with $t \omega$, the transmission function can be described as

$$
T(\omega)=\frac{A}{2}[1+\cos (t \omega+\Delta \varphi)]
$$

Obviously, the transmission function $T(\omega)$ is a standard cosine function. But the variable in $T(\omega)$ is angular frequency $\omega$ not time $t$. According to Fourier synthesis theory, the sinusoidal function is the fundamental condition to compose an arbitrary function. In time domain, an arbitrary signal $S(t)$ can be generated through integrating signals with basic frequency $\omega_{0}$ and different harmonic frequencies $n \omega_{0}$. Similarly, arbitrary frequency spectrum $S(\omega)$ also can be synthesized with basic time $t_{0}$ and several harmonic time $n t_{0}$, which correspond to the basic frequency $\omega_{0}$ and harmonic frequency $n \omega_{0}$ in time domain, respectively.

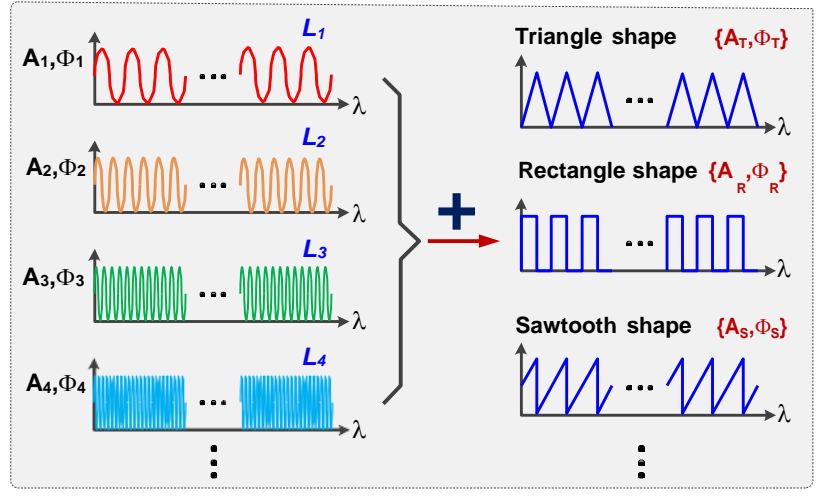

Fig. 1. The schematic diagram of arbitrary spectral synthesis with parallel-connecting HiBi-FLMs. $A_{i}$ : the amplitude of transmission function; $\Phi_{i}$ : the phase of transmission function; $L_{i}$ : the length of PMF.

According to the above analysis, arbitrary spectrum can be synthesized utilizing several parallel-connecting HiBi-FLMs (whose transmission spectra are standard cosine functions) with assigned amplitudes and phases. As shown in left side of Fig. 1, HiBi-FLMs have different free spectrum range (FSR) of the transmission spectra due to different length of PMFs. According to the equation of $t=B L / c$, the basic time $\left(t_{0}\right)$ and harmonic time $\left(n t_{0}\right)$ can be controlled by adjusting the length $(L)$ of PMFs. Besides, the phase and amplitude of each HiBi-FLM can be independently adjusted because of parallel connection. The transmission function $\left(T_{S}\right)$ of the HiBi-FLMs array can be described as

$$
T s(\omega)=A_{S}+\frac{1}{2 n} \sum_{i=1}^{n} A_{i} \cos \left(t_{i} \omega+\Delta \varphi_{i}\right)
$$

where $A_{S}=\left(A_{1}+\ldots+A_{i}+\ldots+A_{n}\right) / 2 n, \Delta \varphi_{i}$ is the phase variation, $A_{i}$ and $\Delta \varphi_{i}$ can be adjusted with PCs, $n$ is the number of the $\mathrm{HiBi}-\mathrm{FLM}, i$ is the sequence number of HiBi-FLM, $t_{i}$ equals to $B L_{i} / c$. Obviously, the transmission function satisfies the Fourier synthesis theory such that arbitrary spectrum $T_{S}$ can be achieved (as illustrated in right side of Fig. 1).

\section{EXPERIMENT AND DISCUSSION}

In order to verify the feasibility of the proposed scheme, four parallel-connecting HiBi-FLMs have been adopted. Figure 2(a) is our experimental setup to synthesize arbitrary optical spectrum. A broadband light source (BBS) is spilt into four branches through a $1 \times 4(25: 25: 25: 25)$ optical coupler $(O C)$ and then all of them are injected into four parallel-connecting HiBi-FLMs. The HiBi-FLM is composed by a 3-dB OC, a PC and a piece of PMF with different length. At last, all transmission spectra of different HiBi-FLMs are integrated by a $4 \times 1$ OC and observed with an optical spectrum analyzer (OSA, Yokogawa, AQ6370D).

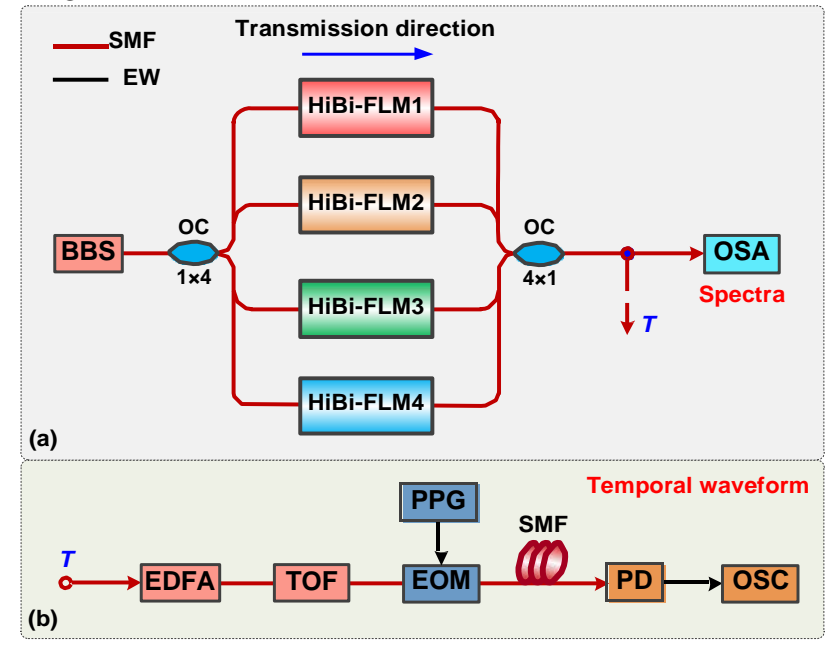

Fig. 2. Experimental setup. (a) The schematic of arbitrary optical spectrum synthesis; (b) the setup of converting optical spectrum into temporal waveform.

\section{A. Optical Spectral Synthesis}

Table 1. The amplitude and phase arrays of the HiBi-FLMs.

\begin{tabular}{c|c|c:c}
\hline Spectral Shape & Triangle & Rectangle & Sawtooth \\
\hline Fiber length & $1-3-5-, 7-m$ & $1-, 3-5-, 7-m$ & $1-, 2-3-, 4-m$ \\
\hline Amplitude ratio & $1,1 / 9,1 / 25,1 / 49$ & $1,1 / 3,1 / 5,1 / 7$ & $1,1 / 2,1 / 3,1 / 4$ \\
\hline Assigned phase & $0,0,0,0$ & $0, \pi, 0, \pi$ & $0,-\pi / 2, \pi, \pi / 2$ \\
\hline
\end{tabular}

In our experiments, three typical spectra including rectangle, triangle and sawtooth shapes were implemented to verify the effectiveness of the proposed approach. Table 1 presents the key parameters of the used HiBi-FLMs, which includes the fiber length of PMF, the corresponding amplitude ratio and the assigned phase of each HiBi-FLM. Here, the PMF is fabricated by YOFC with precise fiber length $( \pm 0.05 \mathrm{~m}$ for each $\mathrm{PMF})$ and its phase modal birefringence $B_{0}$ is about $6.0 \times 10^{-4}$. It should be noted that both the amplitude and phase of HiBi-FLMs can be tuned through adjusting the state of PCs. To adjust the amplitude and phase precisely, theoretical simulation has been 
implemented to guide the adjustment. The simulation is also executed according to Table 1 and it presents the details of each transmission spectrum clearly. The tunable processes are as follows. Firstly, we did theoretical simulations according to the target spectrum and obtained the corresponding transmission spectra, as illustrated in Fig. 3. Then, we obtained the maximum transmission power of the HiBi-FLM1 by adjusting PC1 and recorded the maximum power $\left(A_{l}\right)$ and corresponding phase $\left(\varphi_{1}\right)$. At last, the amplitudes and phases of another three HiBi-FLMs were tuned through adjusting PC according to the phase detail in Fig. 3 and the ratio with $A_{l}$ (shown in Table 1).
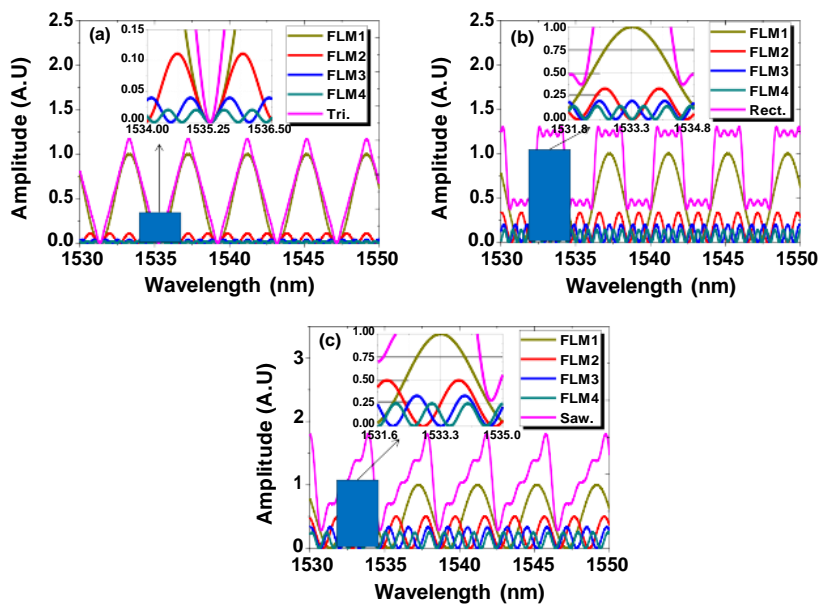

Fig. 3. The theoretical simulations. (a) The triangle, (b) rectangle and (c) sawtooth optical spectrum, respectively.

After elaborately adjusting the amplitude and phase of each HiBi-FLM according to above processes, the experimental results are illustrated in Fig. 4. Besides, theoretical simulations (blue-solid line) have been executed to compare with experimental results (red-solid line). In order to contrast them adequately, all results shown in Fig. 4 are normalized. Figure 4(a) shows the transmission spectrum of the basic HiBi-FLM (consisting of 1-m PMF), which is a standard cosine spectrum. The extinction ratio (ER, defined by the ratio of the maximum and minimum transmission power) and the FSR of the basic HiBi-FLM are $28.95 \mathrm{~dB}$ and $\sim 3.9 \mathrm{~nm}$, respectively. Figure 4(b) shows the synthesized triangle-shaped optical spectrum and its corresponding numerical simulation. It can be observed that the full bandwidth of the triangle-shaped optical spectrum is $~ 3.9$ nm (agreeing well with the FSR in Fig 4(a)) and the ER of the synthesized spectrum reaches up to $21.01 \mathrm{~dB}$. However, the bandwidth and ER cannot estimate the performance completely. Therefore, the goodness of fit (GoF) between the theoretical simulation (blue-solid line) and test result (red-solid line) was introduced to evaluate the conformity and it is $\sim 0.9529$. Here, the GoF is calculated according to the following equation.

$$
R=1-\sqrt{\frac{\sum\left(y-y^{*}\right)^{2}}{\sum y^{2}}}
$$

where $y$ is the test result, $y^{*}$ is the simulation value, $R$ is the GoF. Obviously, the test result is more consistent with the theoretical estimation as $R$ increasing and its maximum value is 1 .

After that, the rectangle- and sawtooth-shaped spectra have also been synthesized with same method. Figure 4(c) shows the rectangle-shaped optical spectrum and the passband of each rectangle shape is $\sim 1.95 \mathrm{~nm}$. The ER of $\sim 5.13 \mathrm{~dB}$ is slightly smaller than the estimation value $(\sim 5.43 \mathrm{~dB})$ and the $\mathrm{GoF}$ between the experimental result and theoretical simulation is 0.9357. Another sawtooth-shaped optical spectrum is depicted in Fig. 4(d) and the full bandwidth of each sawtooth-shaped optical spectrum is $\sim 3.9 \mathrm{~nm}$. The ER and GoF are $\sim 8.01 \mathrm{~dB}$ (the estimated ER is $\sim 8.12 \mathrm{~dB}$ ) and 0.9565 , respectively.

Although the obtained ERs are low in rectangle- and sawtooth-shaped spectra, they are extraordinarily close to theoretical values. Besides, all synthesized spectra are in good agreement with theoretical simulations except for the last two periods (from $\sim 1543 \mathrm{~nm}$ to $1550 \mathrm{~nm}$ ), as illustrated in Fig. 4(b) to Fig. 4(d). That is caused by imprecise lengths of HiBi-FLMs and can be mitigated by using more accurate HiBi-FLMs or longer PMF.
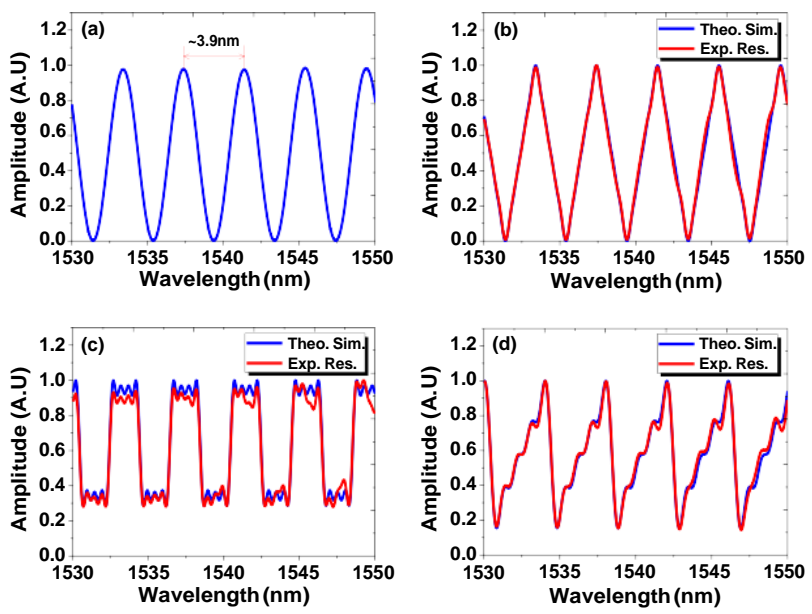

Fig. 4. The comparison of experimental result (Exp. Res.) and theoretical simulation (Theo. Sim.). (a) The transmission spectrum of the basic HiBi-FLM (which is composed by 1-m PMF); (b) triangle-, (c) rectangle- and (d) sawtooth-shaped optical spectrum, respectively.

\section{B. Arbitrary waveform generation}

The proposed optical spectrum synthesis method has many potential applications such as bandpass filter, edge filter and arbitrary waveform generation (AWG) et al. To verify its typical application, temporal AWG have been implemented with the synthesized arbitrary spectra incorporating with FTTM technique. Figure 3(b) is our experimental setup based on FTTM process. The synthesized optical spectrum from Fig. 3(a) acts as the light source. After being amplified and filtered by erbium-doped fiber amplifier (EDFA) and tunable optical filter (TOF, which is used to select one shape in the periodic spectrum) consecutively, the light was intensity-modulated by an electrical optical modulator (EOM). The EOM was drived by a pulse pattern generator $(\mathrm{PPG})$ at a bit rate of $10 \mathrm{Gbit} / \mathrm{s}$ with code of "1100000000". So the repetition period and original

width of the optical pulse are $1 \mathrm{~ns}$ and $\sim 210 \mathrm{ps}$ (including rising and failing edge), respectively. After that, the modulated optical pulses were launched into dispersive element. Here, a 10- $\mathrm{km}$ single mode fiber (SMF) was used as the firstorder linear dispersive element with total group delay dispersion (GDD) value of $170 \mathrm{ps} / \mathrm{nm}$. Lastly, the optical pulses after FTTM process were converted into electrical signal at a 
high-speed photodetector (PD, 20-GHz bandwidth) and collected by an oscilloscope (with sample rate of $40 \mathrm{GS} / \mathrm{s}$ ) with 20-times average, respectively.
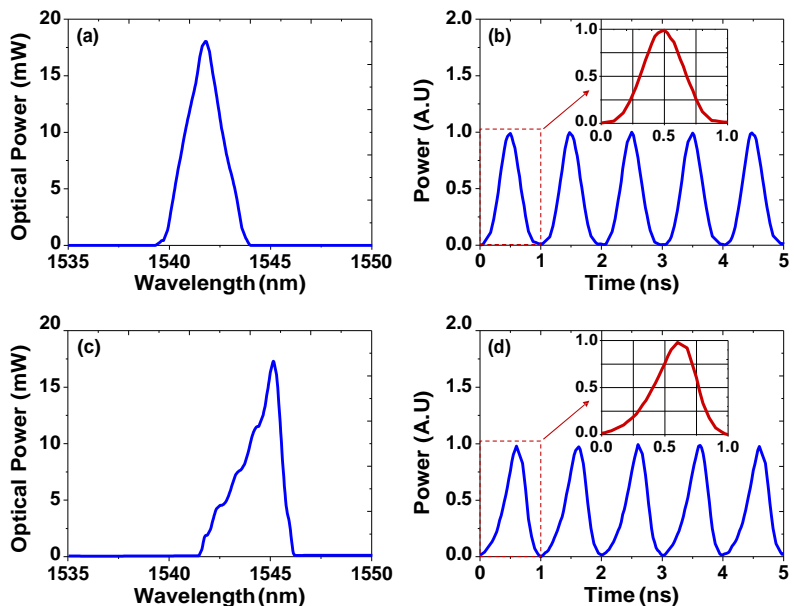

Fig. 5. The generation of the triangle- and sawtooth-shaped waveform. (a) The optical spectrum after passing through TOF; (b) the generated temporal triangle-shaped waveform; (c) the optical spectrum after passing through TOF; (d) the generated temporal sawtooth-shaped waveform with negative ramp.

In AWG experiments, triangle- and sawtooth-shaped temporal waveforms were generated. Figure 5(a) and 5(b) show the measured triangle-shaped optical spectrum after passing through TOF and the generated triangle-shaped temporal waveform, respectively. As illustrated in Fig. 5(b), the repetition period and the width of the triangle pulses are $1 \mathrm{~ns}$ and $\sim 860 \mathrm{ps}$, respectively. That is in good agreement with the theoretical computation since the full bandwidth of the optical spectrum, the GDD and the original pulse width are $\sim 3.9 \mathrm{~nm}$, $170 \mathrm{ps} / \mathrm{nm}$ and $210 \mathrm{ps}$, respectively. Another typical waveform of sawtooth shape has also been generated with same method. Figure 5(c) and 5(d) show the measured optical spectrum before being injected into SMF and the sawtooth-shaped electrical pulse series, respectively. As indicated in the inset of Fig. 5(d), the sawtooth-shaped waveform with negative ramp has been obtained, which is similar to the optical spectrum (as shown in Fig. 5(c)). The generated waveforms have slight distortion due to the non-ideal linear dispersion distribution in wide spectrum. That can be improved with narrow spectrum (realizing with longer PMF) and longer SMF. It should be noted that higher repetition rate $(>1 \mathrm{GHz})$ and narrower pulse width $(<860$ ps) also can be achieved by adopting shorter code length $(<10 \mathrm{bits})$ and shorter fiber length $(<10 \mathrm{~km})$ at the same bit rate of $10 \mathrm{Gbit} / \mathrm{s}$, respectively.

\section{CONCLUSION}

We propose and experimentally demonstrate a novel approach to synthesize arbitrary optical spectrum with parallel-connecting HiBi-FLMs, which is based on Fourier synthesis theory. In order to validate the feasibility of the proposed method, both theoretical simulations and experiment verifications have been implemented. By adopting four HiBi-FLMs with different length of the PMF, three typical spectra of triangle, rectangle and sawtooth shape have been synthesized in our experiment. Although the ERs of some specific spectra (i. e., rectangle- and sawtooth-shaped spectra) are low, they agree well with theoretical values. For the synthesized arbitrary optical spectrum, it can be widely used in many fields such as optical filter, edge filter and arbitrary waveform generation. To verify the application of arbitrary waveform generation, triangle- and sawtooth-shaped electrical pulses with repetition rate of $1 \mathrm{GHz}$ and pulse width of $\sim 860 \mathrm{ps}$ have been generated by incorporating FTTM conversion.

\section{REFERENCES}

[1] X. Fang, and R. O. Claus, "Polarization-independent all-fiber wavelength-division multiplexer based on a Sagnac interferometer," Opt. Lett., vol. 20, no. 20, pp. 2146-2148, 1995.

[2] Y. W. Lee, K. J. Han, B. Lee, and J. Jung, "Polarization-independent all-fiber multiwavelength-switchable filter based on a polarizationdiversity loop configuration," Opt. Exp., vol. 11, no. 25, pp. 3359-3364, 2003.

[3] J. Ge and M. P. Fok, "Passband switchable microwave photonic multiband filter," Sci. Rep., vol. 5, pp. 15882, 2015.

[4] S. Chung, J. Kim, B. A. Yu, and B. Lee, "A fiber Bragg grating sensor demodulation technique using a polarization maintaining fiber loop mirror," IEEE Photon. Technol. Lett., vol. 13, no. 12, pp. 1343-1345, 2001.

[5] C. S. Kim, T. H. Lee, Y. S. Yu, Y. G. Han, S. B. Lee, and M. Y. Jeong, "Multi-point interrogation of FBG sensors using cascaded flexible wavelength-division Sagnac loop filters," Opt. Exp., vol. 14, no. 19, pp. 8546-8551, 2006.

[6] L. R. Chen, "Tunable multiwavelength fiber ring lasers using a programmable high-birefringence fiber loop mirror," IEEE Photon. Technol. Lett., vol. 16, no. 2, pp. 410-412, 2004.

[7] S. Li, K. S. Chiang, and W. A. Gambling, "Gain flattening of an erbium-doped fiber amplifier using a high-birefringence fiber loop mirror," IEEE Photon. Technol. Lett., vol. 13, no. 9, pp. 942-944, 2001.

[8] H. Y. Fu, H. Y. Tam, L. Y. Shao, X. Dong, P. K. Wai, C. Lu, and S. K Khijwania, "Pressure sensor realized with polarization-maintaining photonic crystal fiber-based Sagnac interferometer," Appl. Opt., vol. 47, no. 15, pp. 2835-2839, 2008.

[9] L. Shao, Y. Luo, Z. Zhang, X. Zou, B. Luo, W. Pan, and L. Yan, "Sensitivity-enhanced temperature sensor with cascaded fiber optic Sagnac interferometers based on Vernier-effect," Opt. Commun., vol. 336, pp. 73-76, 2015.

[10] H. Chi, F. Zeng, and J. Yao, "Photonic generation of microwave signals based on pulse shaping," IEEE Photon. Technol. Lett., vol. 19, no. 9, pp. 668-670, 2007.

[11] W. Liu, and J. Yao, "Photonic generation of microwave waveforms based on a polarization modulator in a Sagnac loop," J. Lightwave Technol., vol. 32, no. 20, pp. 3637-3644, 2014.

[12] W. Jin, C. Wang, H. Xuan, and W. Jin, "Tunable comb filters and refractive index sensors based on fiber loop mirror with inline high birefringence microfiber," Opt. Lett., vol. 38, no. 21, pp. 4277-4280, 2013.

[13] M. H. Khan, H. Shen, Y. Xuan, L. Zhao, S. Xiao, D. E. Leaird, A. M. Weiner, and M. Qi, "Ultrabroad-bandwidth arbitrary radio frequency waveform generation with a silicon photonic chip-based spectral shaper," Nat. Photon., vol. 4, no. 2, pp. 117-122, 2010.

[14] H. Dong, Q. Wang, H. Sun, and N. K. Dutta, "Stable 80-GHz short pulse generation using the cascaded polarization-maintaining fiber loop mirrors," IEEE Photon. Technol. Lett., vol. 17, no. 7, pp. 1396-1398, 2005.

[15] J. Ye, L. Yan, W. Pan, B. Luo, X. Zou, A. Yi, and S. Yao, "Photonic generation of triangular-shaped pulses based on frequency-to-time conversion," Opt. Lett., vol. 36, no. 8, pp. 1458-1460, 2011.

[16] C. Wang, and J. Yao, "Chirped microwave pulse generation based on optical spectral shaping and wavelength-to-time mapping using a Sagnac loop mirror incorporating a chirped fiber Bragg grating," J. Lightwave Technol., vol. 27, no. 16, pp. 3336-3341, 2009.

[17] Z. Jiang, C. B. Huang, D. E. Leaird, and A. M. Weiner, "Optical arbitrary waveform processing of more than 100 spectral comb lines," Nat. Photon., vol. 1 , no. 8, pp. 463-467, 2007. 